Dermatologische Zeitschrift. 1925;42:I-IV

\title{
Contents, Vol. 42, 1925
}

Inhaltsverzeichnis.

i Origin alarbeiter. Seite-

Back, Rob., Ein Fall von prak"ánzeröser Dermatose Bowen 26(>

Beck, S. C, Über Neosalvarsanbehandlungdes Lupus vulgaris 253-

Brann, Günther, Beitrag zur Behandlung der weiblichen

Gonorrhoe rait Kollumkapseln nach Pust 27

Brünauer, Stefan Rob., Zur Symptomatologie und Histo-

logie der kongenitalen Dyskeratosen ........ (5

Diet el, F. , Ein Fall von Earzinom auf einem Lupus erythe-

matodes 97"

E1 i a s s o w, Alfred, und Adolf Sternberg. Ein Fall von

Pemphigus foliaceus nebst Bemerkungen über die Todes .

uísache bei Pemphigus . . $\quad .1 / 8.186$

Falkenstein, Fritz. Zosterifo $\pi$ ne Pigmentie $\pi m g$ nach

Sehußverletzung

275

Fay, Ad., undAndr. Kolomah Gáal, Über die Eigeneiter-

behandlung der Ulcus molle·Boubonen, glei < 'hzeitig ein'-:-"“-

Versuch zur Erklärung der Wirkungsweise dieser neuen

Behandlungsmethodë- '...? .285

Fischer, H. , Beitrag zur Recklingbausenschen Krankheit,

(MiÊbildungen .am Auge, besonders die markhaltigen

Nervenfasern der Netzliaut) . - . . 143

Gutmánn, C. , Nochmals' zur Fragc der Amyloidosîs der Haut 76

Heesch, Karl, siehe Hoff, Ferd.

H'off, Ferd., und Karl HeescIi, Über unspezifische

Intrakutantherapie bei Hautkrankheiten . . i . . . 348

Hoffmann, E. , Über Spirozid, Stovarsol und Syphilisschutz 101

Resorzin-Perkutol bei Erythrasma . . . . . . . . 104

Tagung der rheiñ.-westf.' Dermatologen-Vereinigung am

9. November 1924 in Bonn .... . . . . an• ‘.’ ‘. 3t5õ

Keutzër, Fritz. Lupus erythematodes in Verbindúng mit

verhornendem Plàttenepithelkrebs. ."-. . . . . - 38

Klaar, Josef, und Rudolf Rosner, Zui · Fragë der lichen-

ruber-artigen Salvai-sanexantheme und Psoriasis vulgaris

nach Salvarsandermatitis $\quad-127$

Kohl, Franz, siehe Od en thai, Heinrich.

Kolonïan, Andr., siehe Fáy·Gaál, Ad. 
Odenthal, Heinrich, und Franz Kohl, Kritisché Bemerkungen zur Arbeit: ..Die" Gonorrhoebehandlung bei Frauen, besonders die Heilfieberbehandlung mit Milch-injektionen" von Werner G. Rumpe $\Gamma$. . . . . . 352:

IV ' Inhaltsvei·zeichnis.

Seite

$3 / 8$ chreus, Th., Bericht über die 88 . Versammlung deutscher

Naturforscher und Arztc in Innsbruck am 21.-27. Sep

tember $1924 \quad 354$

Siemens, H. W. , Zur Kenntnis des systematisierten Naevus

depigmentosus mit Bemërkungen über die formale Genese

der Naevi 65

Somogyi, S i g m u n d . Die Blutserumlipase bei Hauttuberkulose 342

S t r e m p e 1, Rudolf, und G ui s e p p e A rmu z z i, Zur Histopathologie der Wismutstomatitis mit expeririientellen Bei-

trägcn ....; 1

Wä t z o 1 d, Paul, Beitrag zur Entstehung des Pigments. (Nach

Untersuchungen am menschlichen Auge) 323

Wolf, Max, Leberfunktionsproben bei Lues und ihre Be-

deutung zur Verhütung von Salvarsanschäden . . . . 169

.Zerkowitz, Fritz, Über einen Fall von Lichen ruber mit

bemerkenswerter Schleimhautbeteiligung .....; 282

Gesellschaftsbericlite.

Berliner Dermatologischc Gesellschaft. Sitzungen vom 11. III., 25.I11., 13. und 20. V. 1924, 17. VI. 1924 . 41, 106. 197, 295

Tersamnilungsüdwestdeutscher Dermatologen. Sitzungen vom

22. und 23. III. 1924 in Frankfurt a. M. . - . . . 45

88. Versammlung Deutscher Naturforscher und Ärzte in Inns

bruck am 21.-27. Sept. 1924 . 354

$-2 \lambda$. Jahresversammlung der Deutschen Gesellschaft zur Be-

kämpfung der Geschlechtskrankheiten zu Oberhof in

Thüringen am 29. und 30. IV. 192453

Tagung der rhein.-wcstf. Dermatologen-Vereinigung am 9. Nov.

1924 in Bonn a. Rhein .365

Société Française de Dermatologie et de Syphiligraphie.

Sitzungen vom 13. III., 10. IV. und 8. V. 1924 .... 114

Kiewer Gesellschaft für Haut und Geschlechtskrankheiten.

Sitzungen vom 25. III. und 4. XII. 1923, 3. II. u. 2. III. 1924120

Moskauer Venerologisch- Dermatologische (í esellschaft.Sitzungen

vom 1. IV., 6. V., 3. VI.. 7. X., 4. XL. 2. und 27. XII.

1923210

Periodische Literatur ....... 55, 122, 219, 305, 371

Buchbesprechungen 248, 318

Personalien und Tagesnachricliten 04, 120, 321, 387

Sachregister 388

Namenregister 393 
2 um 70. Geburtstag von Professor S. Ehrmann. Von Erich Hoffmann. Bonn a. Rli 320 